\title{
ZDINY
}

\section{Live-cell studies with the Axio Observer}

\section{The Axio Observer inverted research microscope from Carl Zeiss, available with three different stand formats and a variety of other optional features, gives users a flexible and powerful tool for performing fluorescence microscopy.}

The human genome was decoded in 2001, launching a scientific revolution and prompting a flurry of new questions in the scientific community. Since then, international research teams looking into processes inside and between living cells have been concerned less with the 'what', and more with the 'how' and 'why'. How do molecules or proteins, lipids, enzymes, DNA and RNA function within the cell? How do they interact? And why?

In their search for answers to these questions, researchers have focused their efforts on the most sophisticated microscopic techniques for observation, manipulation and analysis-especially those involving fluorescence. The development of such techniques has become a mission for Carl Zeiss, and one that the company has assigned a name: 'FluoresScience'. For many years, this initiative has helped our company to provide some of the leading microscope systems for applied and basic research, and it has helped researchers to open new pathways of discovery in science.

Now a technical innovation is moving science a step further: the Axio Observer inverted research microscope from Carl Zeiss (Fig. 1). It has been developed for maximum flexibility for use with the live-cell techniques of today and tomorrow, and realized as a fully integrated research platform for cell observation, cell manipulation and cell analysis. It can be expanded in a cost-effective fashion from a basic stand for tissue culture and fluorescence, to one for high-speed, laser-scanning microscopy or microdissection.

\section{Perfect conditions for observation}

Regardless of the approach taken, artifact-free microscopic observation is only possible if the ambient parameters are correct for the cells, and temperature, $\mathrm{pH}$ and $\mathrm{O}_{2}$ concentration are among the parameters that must be regulated for this purpose. Absolute precision is of the essence, particularly for extended experiments. The Axio Observer system can be configured with incubator modules that are perfectly adapted to the exacting demands that must be met for regulating these parameters. The regulation of the incubation conditions is fully automated, and performance is further enhanced by an intelligent stack-and-upgrade concept

\section{Eugen Wehner}

Carl Zeiss Microlmaging GmbH, P.O.B. 4041, 37030 Göttingen, Germany. Correspondence should be addressed to E.W. (micro@zeiss.de).

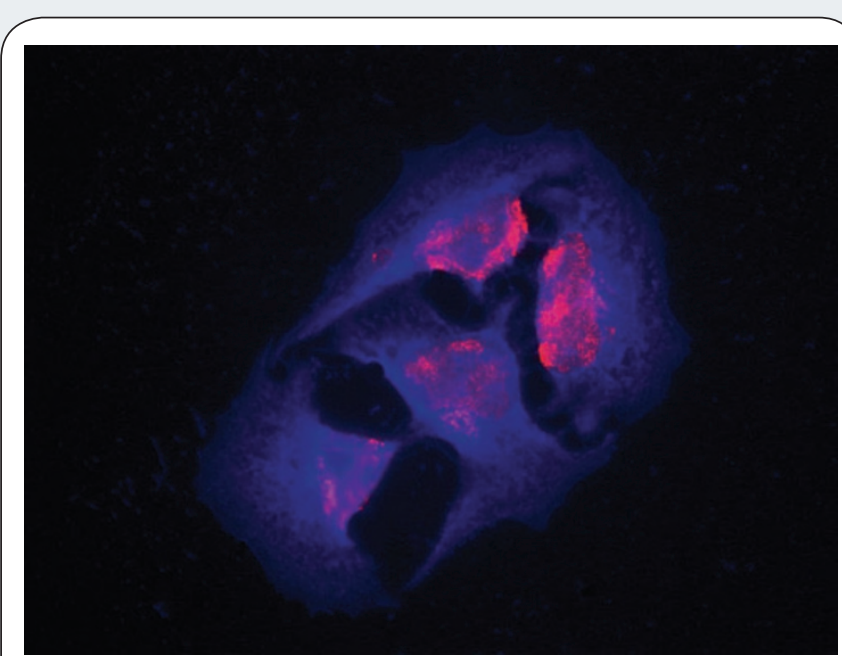

Figure 1 | Live-cell imaging with the Axio Observer. HeLa cells with cytoskeleton (blue) and nucleus (red) labeled with quantum dots.

for the regulatory modules. This overall concept for cell observation is unique and provides for the easy acquisition of all incubation data.

\section{Finding answers through manipulation}

Observation is only one dimension. Often, new insights are only possible via direct communication with the cell and where the cell is forced to respond. Here once again, various approaches are available, and present capabilities are often inadequate.

Laser experiments, for example, can prove to be complex. For example, these could require the targeted bleaching of specific subsets of fluorophores, as with fluorescence recovery after photobleaching experiments. On the basis of how the fluorescence signals are regenerated, a scientist can potentially distinguish between free diffusion, active transport processes or hindered diffusion because of membranes. It is also possible to use a laser to convert cellular proteins or fluorophores from an inactive to an active form via uncaging. If enzymes are uncaged, an entire chain of processes can be activated in the cell at a desired time. What is important in all these processes is simultaneous monitoring with fluorescence or with transmitted light. With its new laser port, Axio Observer is open for all laser applications, and there is 
APPLICATION NOTES

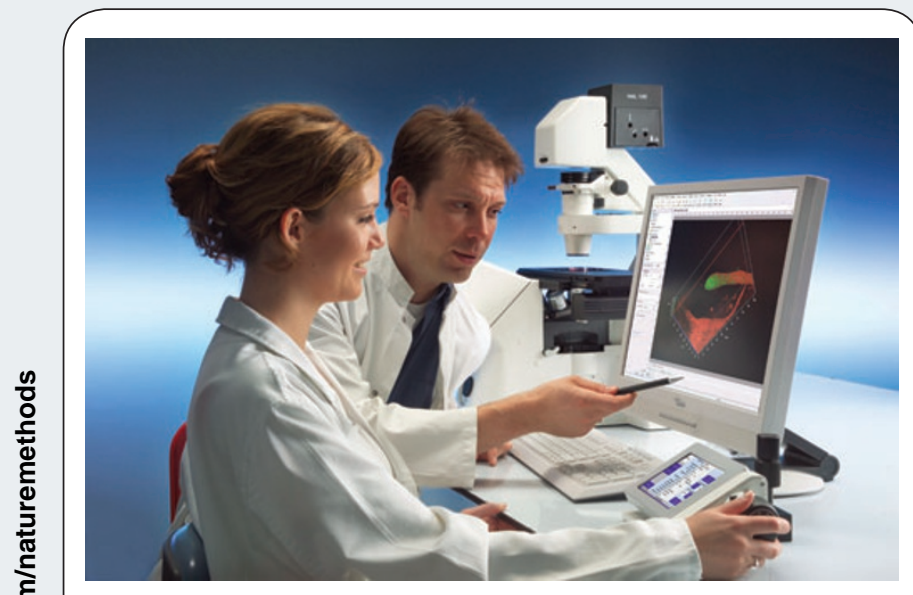

Figure $\mathbf{2}$ Targeted analysis with the AxioVision software suite.

no need for any enlargement of the infinity space, which prevents the reduction in optical quality.

\section{Efficient editing and combination of the raw data in the} analysis

It is essential for raw data to be condensed down to essentials. Only in this way is it possible to make meaningful interpretations and achieve decisive scientific progress. Axio Observer allows this in many different ways: the storage of environmental data within image files, the calculation of emission intensities in ratio experiments during an experiment or through the use of highly advanced downstream modules in our AxioVision microscope software (Fig. 2). These modules include specialized tools for deconvolution, spectral unmixing, colocalization and the detection of image areas in which motion is taking place.

\section{The Axio Observer system}

At all levels, maximum performance is expected from a microscope system, and this is the basis of the Axio Observer system. At the core of the overall system are many new functions, including major improvement of contrast techniques to include a new level of differential interference contrast quality with a clearly homogenous background, an apochromatic fluorescence beam-path with homogenous excitation

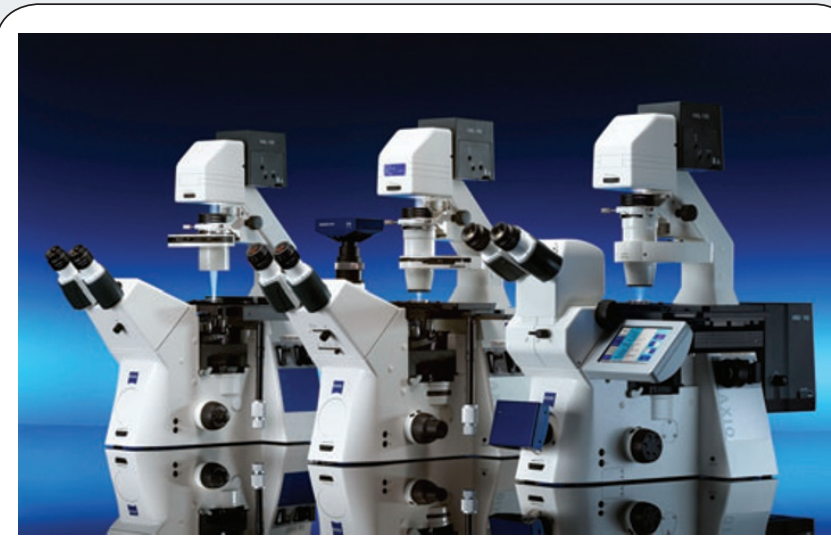

Figure 3 Different configurations of the Axio Observer instrument from Car Zeiss. Axio Observer A1 with a manual stand (left), Axio Observer D1 with a semimotorized stand (middle), and Axio Observer Z1 with a fully motorized stand and including all optional features (right).

for better contrast in the periphery of the field of view, and finally, variable phase-contrast that combines negative and positive phasecontrast in one objective lens. All of these optimizations provide the extra information that can be so important today. These enhancements are rounded off by a completely new operating concept that is effective at all levels. This includes various motorized diaphragms, a $6 \times$ reflector turret that can be equipped without any need for removal, and automatic component recognition for increased safety and ease of use. Another unique optional feature is the touchscreen, thin-film transistor display for the complete operation of the Axio Observer. This can be used as a docking station and can therefore be directly positioned at the PC. The three different stand types available for the Axio Observer-A1, D1 and Z1-cover the entire range of possible applications (Fig. 3).

Last but not least, with the Axio Observer, Carl Zeiss remains true to the pledge and commitment the company made with its FluoresScience initiative: to provide leading microscope systems for applied and basic research that offer maximum contrast and exhaust all technical possibilities for fluorescence applications.

This article was submitted to Nature Methods by a commercial organization and has not been peer reviewed. Nature Methods takes no responsibility for the accuracy or otherwise of the information provided. 